\title{
BMJ Open Design, development and randomised controlled trial of a smartphone application, 'QinTB', for smoking cessation in tuberculosis patients: study protocol
}

\author{
Haoxiang Lin (D) , ${ }^{1}$ Yan Lin, ${ }^{2}$ Yunting Zheng, ${ }^{1}$ Zhao Liu, ${ }^{3}$ Chun Chang ${ }^{1}$
}

To cite: Lin $\mathrm{H}$, Lin Y, Zheng $\mathrm{Y}$, et al. Design, development and randomised controlled trial of a smartphone application, 'QinTB', for smoking cessation in tuberculosis patients: study protocol. BMJ Open 2019;9:e031204. doi:10.1136/ bmjopen-2019-031204

- Prepublication history for this paper is available online. To view these files, please visit the journal online (http://dx.doi org/10.1136/bmjopen-2019031204).

Received 22 April 2019 Revised 28 0ctober 2019 Accepted 30 October 2019

Check for updates

(c) Author(s) (or their employer(s)) 2019. Re-use permitted under CC BY-NC. No commercial re-use. See rights and permissions. Published by BMJ.

${ }^{1}$ Department of Social Medicine and Health Education, School of Public Health, Peking University Health Science Center, Beijing, China

2International Union Against Tuberculosis and Lung Disease, Paris, France

${ }^{3}$ Tobacco Medicine and Tobacco Cessation Center, China-Japan Friendship Hospital, Beijing, China

Correspondence to Dr Chun Chang; changchun@bjmu.edu.cn

Dr Zhao Liu;

Iz|zq2004@126.com

\section{ABSTRACT}

Introduction Providing smoking cessation treatment is an important intervention for tuberculosis (TB) patients. Mobile technologies, such as smartphone applications, have shown promising potential. However, there are few effective applications that could support TB patients in their efforts to quit smoking. To address this problem, we will develop a smartphone application 'QinTB' to help TB patients quit smoking, and we will evaluate the clinical efficacy of this application by using a randomised controlled trial (RCT).

Methods and analysis This is a two-step study. In the first step, we will develop a smartphone application based on an interactive application of the transtheoretical model and protection motivation theory. Then, we will perform an RCT using a two-arm design; a total of 400 patients will be randomly assigned to the application group or the doctors' advice group; both treatments will be 6 months and follow-up will be 12 months; the primary outcome is the biochemically verified 6 month sustained abstinence rate; data will be analysed on an intention-to-treat basis.

Ethics and dissemination This study was approved by the Ethics Committee of Beijing Research Institute for Tuberculosis Control and Prevention. We will disseminate the findings of this study through peer-reviewed publications and conference presentations.

Trial registration number This study was registered in the Chinese Clinical Trial Registry (ChiCTR1900022008) and the stage is Pre-results.

\section{BACKGROUND}

\section{Smoking and tuberculosis}

Tuberculosis (TB) is an airborne infectious disease caused by Mycobacterium tuberculosis; TB remains a major public health problem in most low- and middle-income countries despite great progress over the past decades. In 2015, it was estimated that there were 10.4 million new infections and 1.8 million deaths caused by TB worldwide. ${ }^{12}$

Overwhelming evidence has confirmed that both active smoking and secondhand smoking exposure are associated with an
Strengths and limitations of this study

- 'QinTB', the first smartphone application for smoking cessation in tuberculosis (TB) patients in China, is grounded in transtheoretical model theory and protection motivation theory.

- This is the first trial to evaluate an intervention delivered by mobile phones for smoking cessation in TB patients in China. If the application is found to be effective, it has the potential to reach a large number of people at a low cost.

- Participants must own a personal iPhone to receive the intervention, which means that the sample may not represent a broad socioeconomic range of people.

- Another bias is the impossibility to blind the participants and the different modes of questionnaire administration used (application-based vs paper-based)

increased risk of TB infection. The 2014 Surgeon General's Report concluded that tobacco use was associated with an approximate doubling of the risk of TB infection. ${ }^{3}$ Smokers are reported to have a higher likelihood of positive smears and cavitary pulmonary TB than non-smokers. ${ }^{4}$ Moreover, studies have reported that TB patients who smoke experience delayed access to health services, delayed sputum conversion and an increased risk of unfavourable treatment outcomes and relapse after successful completion of anti-TB treatment. ${ }^{5-8}$

Smoking cessation is essential, but it is not integrated into TB care

It is well established that providing TB patients with effective smoking cessation treatment is an important step for TB care. Smoking cessation would be beneficial to improve not only treatment outcomes but also overall quality of life among TB patients. ${ }^{910}$ 
Therefore, the International Union Against Tuberculosis and Lung Disease (The Union) and WHO have both recommended the integration of tobacco cessation treatment into routine TB care. ${ }^{11}$ The WHO End TB Strategy calls for global action on the co-management of TB and smoking and other comorbidities as part of basic clinical management. ${ }^{12}$

Despite its importance, smoking cessation has not been integrated into routine TB care in China. This is largely due to the fact that TB care is provided in special infectious disease hospitals that are separated from the general care facilities in the Chinese medical system. Shin et al found that Chinese TB patients do not routinely receive effective smoking cessation support during this 'teachable moment', even though TB doctors realise the importance of offering smoking cessation interventions as part of TB treatment. ${ }^{13}$ Lin et al found that the majority of TB patients who smoked were willing to quit and could achieve a better quit rate with effective cessation support in China. ${ }^{14}$

\section{Mobile health technology for smoking cessation among TB patients is feasible but lacks evidence}

Previous studies have confirmed that smartphone application, one of the mobile health technologies, could promote smoking cessation effects and improve health outcomes. Application-based interventions are cost-effective and can proactively reach out to smokers. Support can be offered with or without an internet connection, providing effective in-the-moment help, increasing involvement and tracking progress at any time. ${ }^{15-17}$

Currently, there is neither high-quality evidence nor effective smoking cessation applications on in the market that could support TB patients to quit smoking during this 'teachable moment'. In addition, some limitations have been identified, including the following: not all applications follow international smoking cessation guidelines ${ }^{16}$; the content and theory used in the development process is not clear, and therefore, researchers cannot take advantage of previous experiences ${ }^{18}$ and most studies focus on the short-term impact on smoking cessation rather than longer-term evidence.

\section{OBJECTIVE}

We aim to develop a smartphone application 'QinTB' to support TB patients in their efforts to quit smoking based on behaviour-change theories while taking into consideration the context for the Chinese people. After this, we will conduct a randomised controlled trial (RCT) to verify the efficacy of 'QinTB'. The secondary objective is to identify whether certain patients are more likely to benefit from such application (APP) interventions.

\section{METHODS}

This study will be conducted in two steps. We will first develop a smoking cessation smartphone application
'QinTB' based on the interactive application of the transtheoretical model (TTM) and protection motivation theory (PMT). Both models have been independently applied to health behaviour change interventions. Then, a prospective RCT using a two-arm design will be conducted to assess the efficacy of 'QinTB'.

\section{Step 1: design and development of the smartphone application 'QinTB'}

The design and development processes have three stages.

The first stage is to develop the conceptual and theoretical framework based on the TTM and PMT. TTM is a systematic theory of behaviour change that states that health behaviour is determined on a stage-to-stage basis. ${ }^{19}$ Applied to the 'QinTB' application, the behaviour of smoking cessation is divided into five stages: precontemplation, contemplation, preparation, action and maintenance. For any stage, the specific interventions will be provided through 'QinTB' to strengthen the behaviour change occurrence and/or support to achieve the next stage.

PMT is a systematic theory that states that intention, as the most significant predictor of behaviour, is determined by two processes: threat appraisal and coping appraisal. Threat appraisal is determined by an individual's beliefs about the negative consequences of the health threat (perceived severity), their vulnerability to the negative consequences of the threatened event (perceived susceptibility) and the benefits of the performance of the maladaptive behaviour (intrinsic and extrinsic rewards). Coping appraisal is determined by an individual's beliefs about the effectiveness of the preventative behaviour for the threatened event (response efficacy), confidence in one's ability to perform the preventative behaviour (selfefficacy) and barriers to performance of the preventative behaviour (response costs). ${ }^{20}{ }^{21}$ Applied to 'QinTB', the behavioural intervention will be provided to enhance smokers' perception of the severity of and their susceptibility to negative consequences and decrease smoking intrinsic and extrinsic rewards, aiming to increase threat appraisal. Moreover, a personalised intervention will be provided to enhance self-efficacy and decrease response costs, thus strengthening coping appraisal. The conceptual and theoretical framework for the 'QinTB' application based on the TTM and PMT is shown in figure 1, and the theories underlying the APP design are further described in table 1 .

In the second stage, we will hold several meetings. Experts in smoking cessation, health education, TB, etc, will join the meetings and will provide comments and suggestions for the 'QinTB' content. Then, the study team will revise those sections accordingly.

The last stage is to develop the 'QinTB' by using information technology. There are several important considerations. First, the application should ensure confidentiality of the data. Second, the system needs to be user-friendly with ease for quick data entry. Third, there needs to be a back-end server that stores the data 


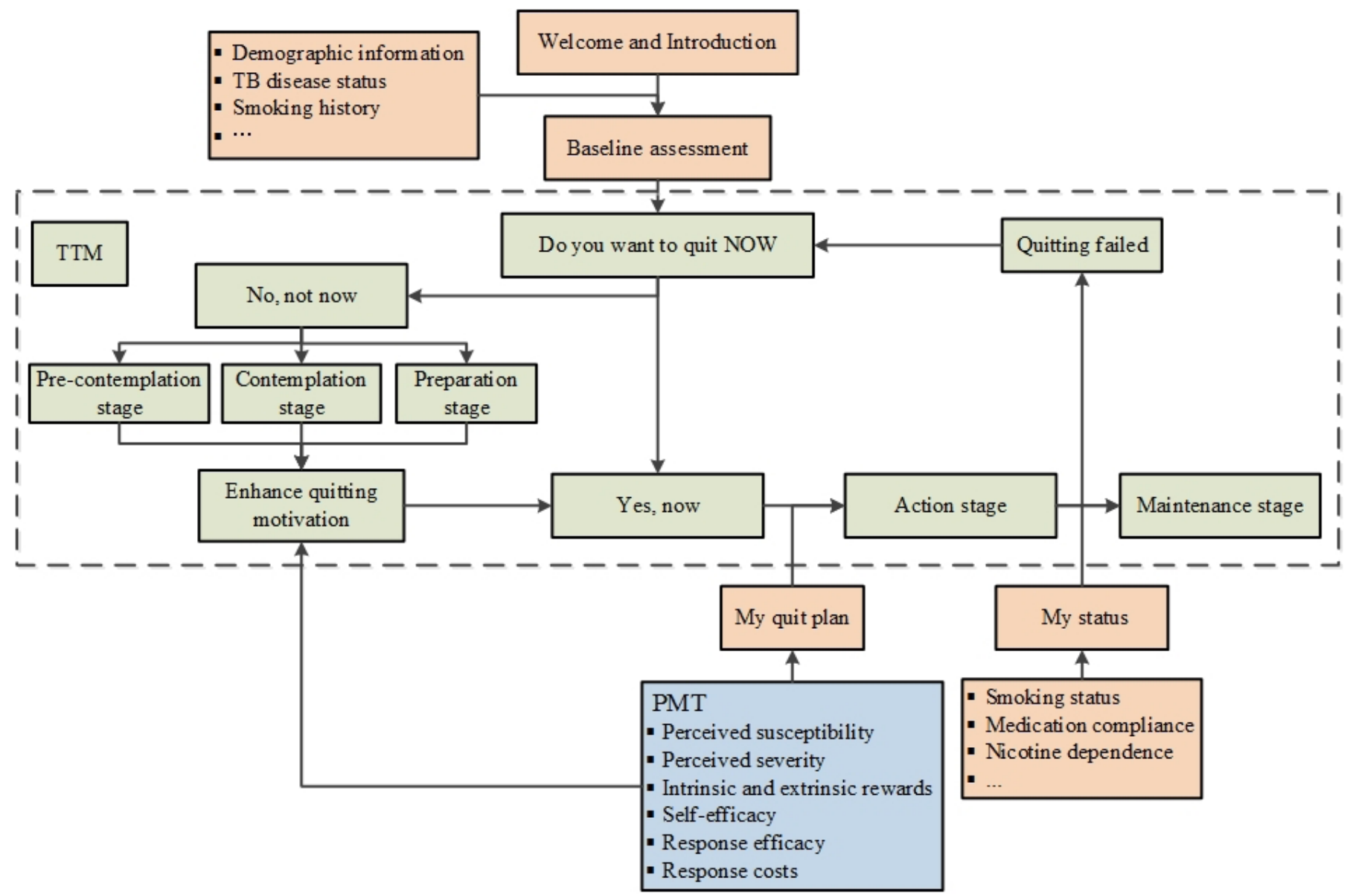

Figure 1 Application structure and features. PMT, protection motivation theory; TB, tuberculosis; TTM,transtheoretical model.

for future analysis. Fourth, it should be usable and adaptable for common operating systems. Finally, the information must be presented in a way that is easily read and interpreted. Therefore, LimeJS, an HTML5 framework for touch screens and desktop browsers, will be chosen as the basis for the development of this application. The MySQL database will be used to track the smokers' data and behaviours. On the server port, with multi-tier architecture, this application will divided into a display layer, a business layer and data layer to improve system reliability. In addition, in view of the possible high number of concurrent requests, container technology will be used to implement the rapid deployment strategy of multiservice instances and a streaming media player server will be established for video and audio playback to ensure fast response under the condition of high concurrent requests.

\section{Step 2: prospective RCT to evaluate the APP-based smoking cessation intervention in TB patients \\ Enrolment procedures and eligibility criteria}

We will recruit patients from Beijing Research Institute for Tuberculosis Control and Prevention and other health institutions if necessary. Newly registered TB patients will be assessed for eligibility and will be invited to participate in this study if they fulfil the following criteria. If the contacted patients are willing to participate, written informed consent will be obtained.

\section{Inclusion criteria}

1. Male, aged 18 to 60 years;

2. Diagnosed as active pulmonary TB based on WHO guidelines: including sputum smear-positive and smear-negative pulmonary TB patients;

3. Daily smokers, with $\geq 10$ cigarettes smoked per day and $\geq 5$ years of smoking duration (we require participants to be daily smokers to exclude smokers who are more likely to quit);

4. Expired air carbon monoxide $\geq 9 \mathrm{ppm}$;

5. Intention to quit smoking at precontemplation stage (not considering quitting in the next 6 months), contemplation stage (considering quitting in the next 6 months, but not in the next 30 days) and preparation stage (willingness to quit smoking within the next 30 days);

6. Own an iPhone;

7. Agree to participate and sign an informed consent form.

\section{Exclusion criteria}

1. Received any smoking cessation treatment within 30 days; 


\section{Table 1 Conceptual and theoretical framework for 'QinTB'}

\begin{tabular}{|c|c|c|}
\hline Theory & $\begin{array}{l}\text { Application } \\
\text { to 'QinTB' }\end{array}$ & Description and example \\
\hline \multirow[t]{2}{*}{ TTM } & $\begin{array}{l}\text { Identify } \\
\text { intention-to- } \\
\text { quit stage }\end{array}$ & $\begin{array}{l}\text { Patients' willingness to quit will be assessed and divided into precontemplation, contemplation, } \\
\text { preparation and action stage. } \\
\text { Example: Do you want to quit smoking now, or later? }\end{array}$ \\
\hline & $\begin{array}{l}\text { Provide } \\
\text { stage- } \\
\text { to-stage } \\
\text { support }\end{array}$ & $\begin{array}{l}\text { Given the systematic relationship between the stages and processes of change, several techniques } \\
\text { will be used to strengthen the behaviour change occurrence and/or to achieve next stage. } \\
\text { Consciousness raising, dramatic relief, environmental reevaluation strategies will be used to reach } \\
\text { the contemplation stage; self-reevaluation strategy will be used to reach the preparation stage; self- } \\
\text { liberation strategy will be used to reach the action stage; helping relationships, counterconditioning, } \\
\text { reinforcement management and stimulation of control strategies will be used to reach the } \\
\text { maintenance stage. } \\
\text { Example: If a smoker is at the stage of precontemplation, the information delivered by the APP } \\
\text { will focus on knowledge about smoking harm (consciousness raising) and how quitting can reduce } \\
\text { secondhand smoke exposures to your family (environmental reevaluation). }\end{array}$ \\
\hline PMT & $\begin{array}{l}\text { Strengthen } \\
\text { cessation } \\
\text { protection } \\
\text { motivation }\end{array}$ & $\begin{array}{l}\text { Personalised interventions will be provided according to demographic characteristic (such as age, } \\
\text { gender, education), social psychological factors (such as personality, social status), structural factors } \\
\text { (such as perception of smoking and disease) and will take into consideration current smokers' } \\
\text { cessation protection motivation level. } \\
\text { Example: If a smoker has lower score of 'perceived severity', the APP will deliver more information } \\
\text { about the harm caused by smoking. }\end{array}$ \\
\hline
\end{tabular}

APP, application; PMT, protection motivation theory; TTM, transtheoretical model.

2. Diagnosis of HIV/AIDS or any mental diseases;

3. Unwilling to participate or unable to finish the study.

\section{Randomisation}

An independent statistician, Dr Huaqing Tan (Guanghua School of Management, Peking University) will oversee the randomisation but will not be involved in the treatment. With a randomised block design and the Fagerström test for nicotine dependence as a stratified factor, eligible participants will be assigned to one of two treatment groups: the APP group or the doctor's advice group. The participants, research staff and physicians will not be blinded to the group allocation.

\section{Baseline assessment}

Research staff will conduct a face-to-face interview with recruited patients in their first visit. The interview will take approximately 30 to $45 \mathrm{~min}$ to complete, and answers will be input into a database by the staff. After completing the entire baseline assessment, participants will be given gift card valued at US\$20. Following completion of this assessment, one of the two interventions will be delivered.

\section{APP group}

The participants in the APP group will be instructed to use 'QinTB' after randomisation. When first opening 'QinTB', participants will be welcomed, and they will be able to read information about the application. Next, participants will input their demographic information and complete an assessment. Then, smokers will be classified into the precontemplation stage, contemplation stage or preparation stage based on their intention to quit, according to TTM theory. For smokers who are at the precontemplation stage, the intervention that could enhance quitting motivation will be provided. For smokers who are at the contemplation stage or preparation stage, a 7 day window will be given to set their quit date. Once participants have set their quit date, they will be sent an automated message confirming their choice and recommending that they remain committed to their chosen date. From this point, they will be instructed to report their status every day, particularly their smoking status, TB medication compliance, etc. In the meantime, approximately three to five video and audio messages regarding the dangers of smoking in patients with $\mathrm{TB}$, withdrawal symptoms and the management of cravings, avoiding potential cues and triggers and the benefits of engaging in a behavioural support programme, etc, will be sent per day. In addition, participants will be encouraged to actively read the information in the 'QinTB' application. All participants will be instructed to use this APP for 6 months.

\section{Doctors' advice group}

The participants in the doctors' advice group will receive doctors' advice about smoking cessation from physicians at the Beijing Research Institute for Tuberculosis Control and Prevention. The doctor's advice is based on the ABC intervention outlined in The Union's Guide Smoking 
Table 2 Standard Protocol Items: Recommendations for Interventional Trials

Follow-up

Enrolment Treatment (month)

$\begin{array}{llllllll}\text { Baseline } & 1 & 2 & 3 & 4 & 5 & 6 & 12\end{array}$

\begin{tabular}{|c|c|c|c|c|c|c|c|c|}
\hline \multicolumn{9}{|l|}{ Enrolment } \\
\hline Eligibility screen & $\mathrm{x}$ & & & & & & & \\
\hline Informed consent & $x$ & & & & & & & \\
\hline Allocation & $x$ & & & & & & & \\
\hline \multicolumn{9}{|l|}{ Interventions } \\
\hline APP group & & $x$ & $x$ & $x$ & $\mathrm{x}$ & $x$ & $x$ & $x$ \\
\hline Doctors' advice group & & $x$ & $x$ & $\mathrm{x}$ & $\mathrm{x}$ & $\mathrm{x}$ & $x$ & $x$ \\
\hline \multicolumn{9}{|l|}{ Assessment } \\
\hline Smoking status & $\mathrm{x}$ & $\mathrm{x}$ & $x$ & $\mathrm{x}$ & $\mathrm{x}$ & $\mathrm{x}$ & $\mathrm{x}$ & $x$ \\
\hline Nicotine dependence & $x$ & $\mathrm{x}$ & $x$ & $x$ & $\mathrm{x}$ & $x$ & $\mathrm{x}$ & $x$ \\
\hline Willingness to quit & $\mathrm{x}$ & $\mathrm{x}$ & $x$ & $\mathrm{x}$ & $\mathrm{x}$ & $\mathrm{x}$ & $\mathrm{x}$ & $x$ \\
\hline Prolonged abstinence & & $\mathrm{x}$ & $\mathrm{x}$ & $\mathrm{x}$ & $\mathrm{x}$ & $\mathrm{x}$ & $\mathrm{x}$ & $x$ \\
\hline Perceived susceptibility & $x$ & $x$ & $x$ & $x$ & $\mathrm{x}$ & $\mathrm{x}$ & $\mathrm{x}$ & $x$ \\
\hline Self-efficacy & $\mathrm{x}$ & $\mathrm{x}$ & $x$ & $x$ & $\mathrm{x}$ & $\mathrm{x}$ & $\mathrm{x}$ & $x$ \\
\hline Response efficacy & $x$ & $x$ & $x$ & $x$ & $x$ & $x$ & $x$ & $x$ \\
\hline Response costs & $\mathrm{x}$ & $\mathrm{x}$ & $x$ & $x$ & $x$ & $x$ & $x$ & $x$ \\
\hline Perceived severity & $\mathrm{x}$ & $x$ & $x$ & $x$ & $\mathrm{x}$ & $\mathrm{x}$ & $\mathrm{x}$ & $x$ \\
\hline $\begin{array}{l}\text { Intrinsic and extrinsic } \\
\text { rewards }\end{array}$ & $\mathrm{x}$ & $x$ & $\mathrm{x}$ & $x$ & $\mathrm{x}$ & $\mathrm{x}$ & $x$ & $x$ \\
\hline TB treatment outcomes & & $x$ & $x$ & $x$ & $\mathrm{x}$ & $\mathrm{x}$ & $x$ & $x$ \\
\hline Utilisation of the APP & & $\mathrm{x}$ & $\mathrm{x}$ & $\mathrm{X}$ & $\mathrm{x}$ & $\mathrm{x}$ & $\mathrm{x}$ & $\mathrm{x}$ \\
\hline
\end{tabular}

APP, application; TB, tuberculosis.

Cessation and Smoke free Environment for TB patients. ${ }^{22}$ In brief, it is a three-step cessation intervention method. The 'A' represents asking (ask patients if they currently smoke and if anyone smokes in their home), the ' $\mathrm{B}$ ' represents brief advice (provide patients brief advice to quit smoking), the ' $\mathrm{C}$ ' represents cessation support (provide patients cessation support to help them to quit and make their home smoke-free). The ABC intervention will be conducted each time patients attend face-to-face interviews with the research staff. The treatment duration for the doctors' advice group is 6 months.

A Standard Protocol Items: Recommendations for Interventional Trials is presented in table 2.

\section{Follow-up visit arrangement}

All participants in the two groups will be instructed to attend face-to-face interviews with research staff 1 month, 2 months, 3 months, 4 months, 5 months, 6 months and 12 months after their quit date. In addition, to minimise the confounding effects of physician-patient communication, the interview in the APP group will be less than $10 \mathrm{~min}$, and the words used during physician-patient communication will be more neutral. After completing the entire study, participants will be given another gift card valued at US\$20.

\section{Primary and secondary outcomes}

The primary outcome is the 6 month sustained abstinence rate, defined as a self-report of smoking no more than five cigarettes from 2 weeks after the designated quit date, validated biochemically by an expired carbon monoxide level of less than 8 ppm at the 6 month follow-up and not contradicted by any previous self-report or validation result. $^{23}$

Secondary outcomes are comprised of four aspects:

1. Health behaviour change:

i. Perceived susceptibility, defined as vulnerability to negative consequences. Participants will be asked two questions, and they will report their agreement from 1 (strongly disagree) to 5 (strongly agree). Higher scores indicate greater perceived susceptibility.

ii. Perceived severity, defined as the seriousness of the negative consequences. Participants will be asked three questions, and they will report their agreement from 1 (strongly disagree) to 5 (strongly agree). Higher scores indicate greater perceived severity.

iii. Intrinsic and extrinsic rewards, defined as the benefits of the performance of a maladaptive 
behaviour. Participants will be asked three questions, and they will report their agreement from 1 (strongly disagree) to 5 (strongly agree). Higher scores indicate greater rewards.

iv. Self-efficacy, defined as confidence in one's ability to successfully perform the preventative behaviour. Participants will be asked two questions, and they will report their agreement from 1 (strongly disagree) to 5 (strongly agree). Higher scores indicate greater self-efficacy.

v. Response efficacy, defined as the negative consequences of the threatened event. Participants will be asked three questions, and they will report their agreement from 1 (strongly disagree) to 5 (strongly agree). Higher scores indicate greater response efficacy.

vi. Response costs, defined as the barriers to performance of the preventative behaviour. Participants will be asked three questions, and they will report their agreement from 1 (strongly disagree) to 5 (strongly agree). Higher scores indicate greater response costs.

2. TB treatment outcomes: treatment compliance, TB cure rate, loss to follow-up, treatment interruption and treatment outcome were not evaluated.

3. Utilisation of the APP: total time of APP utilisation, functions used and self-report preferences (eg, participants' preferences for different components of the APP).

4. Whether an actual quit attempt lasting at least 24 hours was made.

\section{Data management}

Professor Xiao Li from the Chinese Center for Health Education will work with staff of the research team to maintain quality assurance for all data. The process will be checked according to the Standard Protocol Items: Recommendations for Interventional Trials. All the data will be stored and password-protected in the Beijing Research Institute for Tuberculosis Control. All patients' information will be only accessible by personnel participating in the study. For the statistical analysis, all patients' information will be encoded to guarantee that the personal information is unidentifiable during the processing of the data or the reporting of the results. The number of patients who drop out will be reported.

\section{Sample size}

The sample size calculation is based on a formula for comparing rates in two independent groups:

$$
\mathbf{N}=\frac{\left[Z_{\alpha} \sqrt{2 \bar{P}(1-\bar{P})}+Z_{\beta} \sqrt{P_{1}\left(1-P_{1}\right)+P_{2}\left(1-P_{2}\right)}\right]^{2}}{\left(P_{1}-P_{2}\right)^{2}}
$$

$\alpha$ is the probability of type I error (significance level) with a two-sided test threshold of $0.05 ; \beta$ is the probability of type II error (1-power of the test) with a one-sided test threshold of 0.2; P1 is the smoking quit rate in the group of patients undergoing doctors' intervention, which was expected to be $35 \%$; $\mathrm{P} 2$ is the smoking quit rate in the group with the mobile phone intervention, which is expected to be $50 \%$ and $\bar{P}$ is the average rate of $\mathrm{P} 1$ and $\mathrm{P} 2$ provided that each group has and equal sample size. According to the formula, the sample size was calculated to be 170 for each group. After an attrition rate of $20 \%$ was taken into consideration, 200 were determined as the sample size for each group.

\section{Statistical analysis}

All data will be analysed using SPSS V.21.0. The level of bilateral significance for all statistical tests will be established at $\alpha=0.05$.

The demographic characteristics, educational background, tobacco use information and smoking cessation stage according to the TTM at baseline will be compared between the two intervention groups using t-tests and Fisher's exact tests.

Data will be analysed on an intention-to-treat (ITT) basis. In accordance with the ITT principle, all patients will be included in the analysis, independent of the groups to which they are randomly assigned and regardless of whether they complete the treatment. Participants who breach the protocol (taking cessation medication or receiving other cessation treatment) will be followed-up as part of their originally assigned group and classified according to their smoking status consistent with the other participants. Participants who are lost to follow-up or do not provide cessation outcome data will be classified as not being abstinent in the analysis.

Logistic and linear regression analyses will be used to compare the effects of two interventions on smoking cessation outcomes, the change of smoking-related health awareness and TB treatment outcomes. All the regression results will be presented as ORs with $95 \%$ CIs or the ratio of geometric means.

\section{Ethics and dissemination}

We will disseminate the findings of this study through peer-reviewed publications and conference presentations.

\section{Patient and public involvement}

This study had no patient involvement. Patients were not invited to comment on the study design and were not consulted to develop patient-relevant outcomes or interpret the results. They were also not invited to contribute to the writing or editing of this document for readability or accuracy.

\section{CONCLUSION}

To our knowledge, this is the first study to develop and evaluate an APP-based intervention for smoking cessation in TB patients in China. The main novelty of our study is the integration of new technologies into behaviourchange therapy and traditional health education techniques. If proven effective, this APP-based intervention 
could make smoking cessation available to a much larger population, including patients with limits for accessing face-to-face treatment.

Acknowledgements We would like to thank Professor Huaqing Tan for his contribution in the randomisation process and Professor Xiao Li for his valuable advice on data quality assurance. We also thank $\mathrm{Dr}$ Bo Xue for his technical support on smartphone APP development. In addition, sincere appreciation is extended to Anne Jones, Technical Advisor, International Union Against Tuberculosis and Lung Disease, for her proofreading skills.

Contributors $\mathrm{CC}$ and ZL should be considered co-corresponding authors. HL and ZL drafted the manuscript; ZL and YL participated in the conception and design of the study; CC, YL and YZ made critical manuscript revisions. All authors approved the final version of the clinical trial protocol.

Funding The authors have not declared a specific grant for this research from any funding agency in the public, commercial or not-for-profit sectors.

Competing interests None declared.

Patient consent for publication Not required.

Ethics approval This study was approved by the Ethics Committee of Beijing Research Institute for Tuberculosis Control and Prevention.

Provenance and peer review Not commissioned; externally peer reviewed.

Open access This is an open access article distributed in accordance with the Creative Commons Attribution Non Commercial (CC BY-NC 4.0) license, which permits others to distribute, remix, adapt, build upon this work non-commercially, and license their derivative works on different terms, provided the original work is properly cited, appropriate credit is given, any changes made indicated, and the use is non-commercial. See: http://creativecommons.org/licenses/by-nc/4.0/.

ORCID iD

Haoxiang Lin http://orcid.org/0000-0003-2952-0299

\section{REFERENCES}

1 World Health Organization. Global tuberculosis report 2018. Geneva, Switzerland: WHO, 2018.

2 Wang X-N, He T-L, Geng M-J, et al. Prevalence of and risk factors for tuberculosis among healthcare workers in Chinese tuberculosis facilities. Infectious Diseases of Poverty 2018;7.

3 U.S. Department of Health and Human Services. The health consequences of smoking-50 years of progress: a report of the surgeon General. Atlanta: Office on smoking and health, 2014.

4 Chen-Yuan C, Singh BT. Should tobacco control intervention be implemented into tuberculosis control program? Expert Rev Respir Med 2018:1-3.

5 Bam TS, Enarson DA, Hinderaker SG, et al. Longer delay in accessing treatment among current smokers with new sputum smear-positive tuberculosis in Nepal. int $j$ tuberc lung dis 2012;16:822-7.

6 Leung CC, Yew WW, Chan CK, et al. Smoking adversely affects treatment response, outcome and relapse in tuberculosis. Eur Respir J 2015;45:738-45.

7 Leung CC, Li T, Lam TH, et al. Smoking and tuberculosis among the elderly in Hong Kong. Am J Respir Crit Care Med 2004;170:1027-33.

8 Abal AT, Jayakrishnan B, Parwer S, et al. Effect of cigarette smoking on sputum smear conversion in adults with active pulmonary tuberculosis. Respir Med 2005;99:415-20.

9 Awaisu A, Nik Mohamed MH, Mohamad Noordin N, et al. The SCIDOTS project: evidence of benefits of an integrated tobacco cessation intervention in tuberculosis care on treatment outcomes. Subst Abuse Treat Prev Policy 2011;6.

10 Awaisu A, Haniki Nik Mohamed M, Noordin N, et al. Impact of connecting tuberculosis directly observed therapy short-course with smoking cessation on health-related quality of life. Tob Induc Dis 2012;10:2-9.

11 Whitehouse E, Lai J, Golub JE, et al. A systematic review of the effectiveness of smoking cessation interventions among patients with tuberculosis. Public Health Action 2018;8:37-49.

12 World Health Organization. The end TB strategy. Geneva, Switzerland: WHO, 2014.

13 Shin SS, Xiao D, Cao M, et al. Patient and doctor perspectives on incorporating smoking cessation into tuberculosis care in Beijing, China. int $j$ tuberc lung dis 2012;16:126-31.

14 Lin Y, Wang L-X, Qiu L-X, et al. A smoking cessation intervention among tuberculosis patients in rural China. Public Health Action 2015:5:183-7.

15 Bricker JB, Mull KE, Kientz JA, et al. Randomized, controlled pilot trial of a smartphone APP for smoking cessation using acceptance and commitment therapy. Drug Alcohol Depend 2014;143:87-94.

16 Buller DB, Borland R, Bettinghaus EP, et al. Randomized trial of a smartphone mobile application compared to text messaging to support smoking cessation. Telemedicine and e-Health 2014;20:206-14.

17 Shahab L, McEwen A. Online support for smoking cessation: a systematic review of the literature. Addiction 2009;104:1792-804.

18 Michie S, Brown J, Geraghty AWA, et al. Development of StopAdvisor: a theory-based interactive Internet-based smoking cessation intervention. Trans/Behav Med 2012;2:263-75.

19 Prochaska JO, Velicer WF. The transtheoretical model of health behavior change. Am J Health Promot 1997;12:38-48.

20 Bashirian S, Barati M, Mohammadi Y, et al. An application of the protection motivation theory to predict breast self-examination behavior among female healthcare workers. Eur J Breast Health 2019;15:90-7.

21 Malmir S, Barati M, Khani Jeihooni A, et al. Effect of an educational intervention based on protection motivation theory on preventing cervical cancer amongMarginalized women in West Iran. Asian Pac J Cancer Prev 2018;19:755-61.

22 Bissell K, Fraser T, Chiang C-Y, et al. Smoking cessation and smokefree environments for tuberculosis patients. Paris, France: International Union Against Tuberculosis and Lung Disease, 2010.

23 West R, Hajek P, Stead L, et al. Outcome criteria in smoking cessation trials: proposal for a common standard. Addiction 2005;100:299-303. 